\title{
Genetic, Comparative Genomic, and Expression Analyses of the Mc1r Locus in the Polychromatic Midas Cichlid Fish (Teleostei, Cichlidae Amphilophus sp.) Species Group
}

\author{
Frederico Henning • Adina Josepha Renz * \\ Shoji Fukamachi $\cdot$ Axel Meyer
}

\begin{abstract}
Natural populations of the Midas cichlid species in several different crater lakes in Nicaragua exhibit a conspicuous color polymorphism. Most individuals are dark and the remaining have a gold coloration. The color morphs mate assortatively and sympatric population differentiation has been shown based on neutral molecular data. We investigated the color polymorphism using segregation analysis and a candidate gene approach. The segregation patterns observed in a mapping cross between a gold and a dark individual were consistent with a single dominant gene as a cause of the gold phenotype. This suggests that a simple genetic architecture underlies some of the speciation events in the Midas cichlids. We compared the expression levels of several candidate color genes Mclr, Ednrb1, Slc45a2, and Tfapla between the color morphs. Mc1r was found to be up regulated in the gold morph. Given its widespread association in color evolution and role on melanin synthesis, the $M c 1 r$ locus was further investigated using sequences derived from a genomic library. Comparative analysis revealed conserved synteny in relation to the majority of teleosts and highlighted several previously unidentified conserved non-coding elements (CNEs) in the upstream and downstream regions in the vicinity of Mclr. The identification of the CNEs regions allowed the comparison of sequences from gold and dark specimens of natural populations. No polymorphisms were found between in the population sample and $M c 1 r$ showed no linkage to the gold phenotype in the
\end{abstract}

F. Henning - A. J. Renz - S. Fukamachi - A. Meyer (ख) Lehrstuhl für Zoologie und Evolutionsbiologie, Fachbereich Biologie, Universität Konstanz, 78457 Konstanz, Germany e-mail: axel.meyer@uni-konstanz.de

F. Henning

e-mail: frederico.henning@uni-konstanz.de mapping cross, demonstrating that it is not causally related to the color polymorphism in the Midas cichlid.

Keywords Conserved non-coding elements - CNEs Comparative genomics . Fish body coloration

Melanocortin-1 receptor - Bacterial artificial chromosome

\section{Introduction}

Animal color patterns often evolve as adaptations to environmental surroundings, in which it frequently involves the evolution of cryptic coloration. Sexual selection acting on color traits can, however, oppose the effects of natural selection and lead to the evolution of conspicuous coloration (Endler 1980). Specifically in cichlid fish, color probably represents an important cue for assortative mating and is related to the establishment and maintenance of reproductive isolation and, hence, speciation (Kocher 2004; Meyer 1993; Seehausen et al. 2008).

The genus Amphilophus includes the Midas cichlid species complex (so far nine species have been formally described), which is distributed throughout the crater lakes in Nicaragua. Several, but not all of these species show a color polymorphism, with dark and gold morphs. Dark individuals have a barred pattern with vertical black bars that are intensified during social interactions, such as during mating behavior and territorial defense. It has been shown that the Midas cichlid species complex has diverged sympatrically at least twice in the Nicaraguan crater lakes (Barluenga et al. 2006; Elmer et al. 2009). In addition to morphological differentiation associated with ecological divergence, color polymorphism has led to significant sub-population differentiation according to color in at least one species possessing the gold polymorphism due to assortative mating between 
gold and dark morphs (Elmer et al. 2009). Gold animals display dark coloration typically less than 1 year of age when they undergo color change, probably through melanophore cell death (Dickman et al. 1988).

The Midas cichlid complex represents, therefore, an exceptionally suited model for the study of origin and maintenance of reproductive isolation in the absence of geographical isolation through the evolution of ecological specialization and color-based assortative mating. The exact mechanism of color change and genes involved are, however, still unknown.

Color patterning in fish involves six types of pigment cells, melanophores (dark pigment), xanthophores (yellow pigment), erythrophores (red pigment), leucophores (white pigment), cyanophores (blue pigment), and iridophores (silvery pigment) (Fujii 2000). The interaction of these cell types, mainly through migration and differential rate of survival and proliferation, generate several whole body tones and patterns (stripes and dots, etc.,). A number of genetic pathways have been described in model fishes such as zebrafish and medaka (Parichy 2003). Some genetic variants of these established pigmentation genes have also been found to be associated to color differences in natural populations (Salzburger et al. 2007; Miller et al. 2007). It has been shown that Teleosts have retained several color genes as duplicates following the fish specific genome duplication (Braasch et al. 2007).

The melanocortin receptor 1 gene $(M c l r)$ encodes a transmembrane receptor member of the G-protein (rhodopsin) gene family. The melanocrotin-1 receptor binds to melanocortins, which are pituitary hormones such as melanophore (or melanocyte)-stimulating hormones (MSHs) and adrenocorticotropin (ACTH) and in mammals to other antagonists, such as the agouti signaling protein (ASIP) and the agouti-related protein (AGRP) which activates the cAMP pathway. Mutations in the $M c l r$ coding region, sometimes single amino acid substitutions, associated with changes in body coloration have been described in several taxa such as birds (Mundy 2005), reptiles (Rosenblum et al. 2004), mouse (Hoekstra et al. 2006), and even extinct taxa (Lalueza-Fox et al. 2007; Rompler et al. 2006). The $M c 1 r$ locus is extremely variable in the human population, where it can display more than 30 alleles and is involved in phenotypes such as red hair, freckles, and a multitude of pigmentation disorders (Healy et al. 2001). In several other mammalian species, it can result in different coat and skin color patterns ranging from dark dominant effects (in case of dominant ligand independent activity) to lighter-colored phenotypes, which tend to result from recessive (loss-of-function) mutations (Garcia-Borron et al. 2005). It is known that MSHs and ACTHs are also involved in physiological color changes in teleosts (Fujii 2000; Richardson et al. 2008).
The $M c l r$ gene coding region has recently been characterized in teleosts, and similarly to other vertebrates, it consists of a single-copy, single-exon gene which contains seven conserved trans-membrane regions (Logan et al. 2003; Selz et al. 2007). Expression levels were found to be similar in all tissues in medaka and stickleback, however, restricted to brains, eyes, skin, and testis in zebrafish (Selz et al. 2007). It was shown recently by genetic mapping and functional assays that the $M c l r$ locus is involved with a recessive brown phenotype, affecting eye color, number, and size of melanophores on the body of the Mexican cave tetra (Astyanax mexicanus). The alleles causing this phenotype have arisen independently in several populations, through the fixation of different mutations in the $\mathrm{Mclr}$ locus. In some populations, however, it was not possible to find mutations in the coding region which suggests the existence of regulatory mutations (Gross et al. 2009).

In this study, we compare expression of a set of candidate pigment genes (Mc1r, Ednrb1, Slc45a2, and Tfap2a) in gold and dark color morphs of the Midas cichlid ( $\mathrm{Am}$ philophus citrinellus). We further analyze sequence data obtained from a bacterial artificial chromosome (BAC) containing the $M c l r$ gene from a genomic library of $A$. citrinellus and use comparative genomic methods to identify conserved non-coding intergenic regions flanking the $M c 1 r$. We then test its causal involvement in the polychromatism in the Midas cichlid species complex by means of sequence comparison of both coding and non-coding regions between dark and gold morphs of Amphilophus xiloaensis and also through analysis of the segregation of the color phenotypes and two Mclr single nucleotide polymorphism (SNP) alleles.

\section{Materials and Methods}

\section{Reverse Transcriptase PCR Using Candidate Genes}

Total RNA was extracted using Trizol from skin and fin tissues of four dark and five gold specimens of A. citrinellus reared in the research animal facility of the University of Konstanz. Quantification was done using spectrophotometry and agarose gel fractioning. Genomic DNA was digested by DNase I recombinant, RNase-free (Roche). Reverse transcription was carried out using oligo dT primers and SuperScript III reverse transcriptase (Invitrogen) at $50^{\circ} \mathrm{C}$ for $2 \mathrm{~h}$.

Candidate genes were chosen based on published results conducted in zebrafish (Parichy 2006) and medaka (Fukamachi et al. 2001) and vertebrates in general (Hoekstra 2006). The following genes and primers were used: $\mathrm{Mclr}$ (melanocortin 1 receptor) $5^{\prime}$-ctctcctttttgtgcaccatc and $5^{\prime}$ gatgacagcattgtcggtgt; Ednrbl (endothelin receptor B) 
$5^{\prime}$-aargaytggtggctkttcag and $5^{\prime}$-gakgccatgttgatsccaat, Slc45a2 (solute carrier family 45 , member 2) $5^{\prime}$-ctgggmggagcwtgtgg ttacand $5^{\prime}$-tggcttcwccsacrgcwgagaa and Tfap $2 a$ (transcription factor AP-2 alpha) $5^{\prime}$-caaagagttcacrgacctgctg and $5^{\prime}$-ctttct gtgettctcrtctttgtc. $\beta$-Actin was used as control $\left(5^{\prime}\right.$-atgtgya arcgiggittygc and $5^{\prime}$-gaytcrtcrtaytcytgytt). RT-PCRs were conducted using the reverse-transcribed and non-reversetranscribed RNAs and $10 \mathrm{ng}$ of genomic DNA as templates. The products obtained were fractioned in agarose-gels and sequenced for verification.

Densitometric analysis was carried out by measuring the area of the bands in Image $J$ (available at http://rsbweb. nih.gov/ij/download.html). The measurements were normalized by the control gene, $\beta$-Actin, and the values plotted using $\mathrm{R}$ (www.r-project.org).

\section{Screening of the BAC Library}

The following nested degenerate $M c l r$ primers for genomic PCR were used: outer-f: $5^{\prime}$-gyyggtrgaraacatcytgg, outer-r: $5^{\prime}$-caaagcammasgaacamagra, inner-f: $5^{\prime}$-sgcggay cgytayatcacca, and inner-r: 5'-tcctgretscggtavgcgta (P. Bunje, unpublished). After direct sequencing of the product and verification of its phylogenetic position with relation to other melanocortin receptors (data not shown; see Fig. 3) the partial Mclr fragment of Midas cichlid was purified twice using QIAquick Gel Extraction Kit (Qiagen) and labeled using NEBlot Phototope Kit (New England Biolabs). Hybridization was performed on our high-density BAC grid filters (unpublished) using Phototope-Star Detection Kit (New England Biolabs). Chemiluminescence from positive clones was detected using CDP-Star, readyto-use (Roche) on Hyperfilm ECL (GE Healthcare).

\section{Shotgun Sequencing and Gap Filling}

We isolated BAC DNA from one of the positive clones (086D13) using QIAGEN Plasmid Midi Kit (Qiagen), and separated its insert from the vector by NotI digestion and pulse-field gel electrophoresis. The insert DNA was electro-eluted into a Spectra/Por dialysis tube (Spectrum Laboratories) and retrieved by isopropanol precipitation. For shotgun-library construction, the insert DNA was sonicated into $\sim 2 \mathrm{~kb}$ fragments, blunt-end-repaired with T4 DNA polymerase, 5'-phosphorelated with T4 DNA kinase, purified by phenol-chloroform, and inserted into SmaI site of $5^{\prime}$-dephosphorelated pUC19 vector using T4 DNA ligase. The products were used to transform One Shot TOP10 Chemically Competent $E$. coli (Invitrogen) and the cloned inserts were amplified by colony PCR using M13 primers. We prepared sequencing templates by treating the products with exonuclease $I$ and temperature-sensitive alkali phosphatase, on which sequencing reactions were performed using BigDye Terminator v3.1 Cycle Sequencing Kit (Applied Biosystems). We used 3100 or 3130xl Genetic Analyzer (Applied Biosystems) for electrophoresis and peak detection.

Reads were assembled into contigs using SeqMan II (DNAstar). In order to fill gaps between the contigs, primer walking was conducted using primers typically designed at $50 \mathrm{bp}$ from the ends of each contig. BAC DNA was isolated using the Qiagen Plasmid Midi Kit following manufacturer's recommendations. Direct sequencing of the gaps was performed using BigDye Terminator v3.1 and $1 \mu \mathrm{g}$ purified BAC DNA as template in a final volume of $10 \mu \mathrm{l}$ in 45 labeling cycles. Gap filling and final assembly was performed using Sequencher v.4.2.

\section{Phylogenetic Analysis}

We collected amino acid sequences of melanocortin receptors $(M c 1 r-M c 5 r)$ for representative vertebrates (human, mouse, chicken, fugu, and zebrafish) from the Entrez Gene database at NCBI, which were aligned using the ClustalW2 program at EMBL-EBI. The tree was made using MrBayes (version 3.1.2) using the $\mathrm{JTT}+\mathrm{G}$ model as suggested by the ProtTest software (version 1.4).

\section{Annotation and Comparative Genomic Analysis}

Genomic sequences spanning $100-200 \mathrm{~kb}$ up and downstream of the Mclr locus were obtained from the following genomes: Takifugu rubripes v.4 (Scf.14:1604290:1905192), Tetraodon nigroviridis v.8 (Chr.5:4741940:5144896), Danio rerio v.7 (Chr.8:25100000:25600000), Oryzias latipes (Chr.3:20709004:21009766), and Gasterosteus aculeatus (Grp.2:11864082:12083744) using the Ensemble Genome Browser (www.ensembl.org). These sequences were annotated manually through the comparison to the $M u s$ musculus protein database in Genbank using the Blastx algorithm. Annotated genomic sequences from Homo sapiens Build 36.3 (Chr.16:88260000:88760000) and Mus musculus Build 37.1 (Chr.8:125859000:126059000) were obtained from the NCBI Map Viewer interface (www.ncbi. nih.gov/mapview).

The $M c I r$ and surrounding intergenic regions, totaling $19,179 \mathrm{bp}$ of the Midas cichlid sequence, from the abovementioned taxa were aligned using the mLAGAN algorithm using mVista (http:/genome.lbl.gov/vista) with anchoring to translated sites.

Comparison of Mclr Alleles Between Dark and Gold Populations in Field Samples

The entire coding region (978 bp) of $M c 1 r$ as well as one upstream $(37 \mathrm{bp})$ and two downstream ( $31 \mathrm{pb}$ and $16 \mathrm{bp}$ ) 
conserved elements were PCR amplified, labeled with BigDye Terminator v.3.1 and sequenced. Sequences obtained were analyzed manually and aligned in the Sequencher software. A total of 30 adult specimens were used, 15 of which of the dark morph and 15 of the gold morph of (A. xiloaensis). These samples were collected in lake Xiloa, Nicaragua in 2007 by T. Lehtonen, K. Elmer and A. Meyer.

The entire $M c l r$ coding region (CR) was obtained by assembling the sequences obtained with two primer pairs, CR1: $5^{\prime}$-aaatgaccaacgggtctctg and $5^{\prime}$ - gatgacagcattgtcggtgt; and CR2: $5^{\prime}$-ctctcctttttgtgcaccatc and $5^{\prime}$-gecgaagcaaaaagaac aca. The $\mathrm{CNEs}$ were obtained with primer pairs designed approximately $200 \mathrm{bp}$ upstream and downstream of the target sequences, namely CNE1: $5^{\prime}$-ctccaagcatttcactcttcg and $5^{\prime}$-acttatggggcatgttcagg; CNE2 $5^{\prime}$-agactgcagtttctcctggaa and $5^{\prime}$-ttagggcaggataagggattt and CNE3: $5^{\prime}$-gatgtagcttttaacg gagca and $5^{\prime}$-ccctgaaaaacgttaagttgg (see Fig. 1).

Segregation Analysis Between the Gold Phenotype and $M c 1 r$ Locus

We used $F_{2}$ intercrossed full-siblings of one dark and one gold grandparents cross of the Meyer-Lab of University of Konstanz. We collected fin clips only from Gold $\mathrm{F}_{2} \mathrm{~S}$ (because dark ones may turn into gold at later stages; see Introduction) and their genomic DNAs were extracted by the phenol-chloroform method. Using a pairs of primers (f: $5^{\prime}$-agcatcctgtaaactacggagacag, r: $5^{\prime}$-ggtgtaacgcaataataaataaggttacaac), we PCR amplified a genomic fragment that covers the entire $M c 1 r$-coding region ( $87 \mathrm{bp}$ of $5^{\prime}$-flanking, $978 \mathrm{bp}$ of the coding, and $226 \mathrm{bp}$ of $3^{\prime}$-flanking sequences) and directly sequenced the product to genotype the SNP.

\section{Results}

\section{Reverse Transcriptase PCR Using Candidate Genes}

Expression of all four color genes was higher in the fins compared to scale tissues. The former tissue was used for between-morph comparisons. Mclr was found to be up regulated and EdnrbI and Tfap2a down regulated in the gold morph (Fig. 1). We focused further on $M c 1 r$, following the previously reported involvement of this gene in pigment evolution of other vertebrates and fish.

BAC Clone Sequencing, Annotation, and Comparative Genomic Analysis

Screening of the BAC library revealed three positive clones, one of which was sequenced. The sequence obtained was used to construct a phylogenetic tree with the a

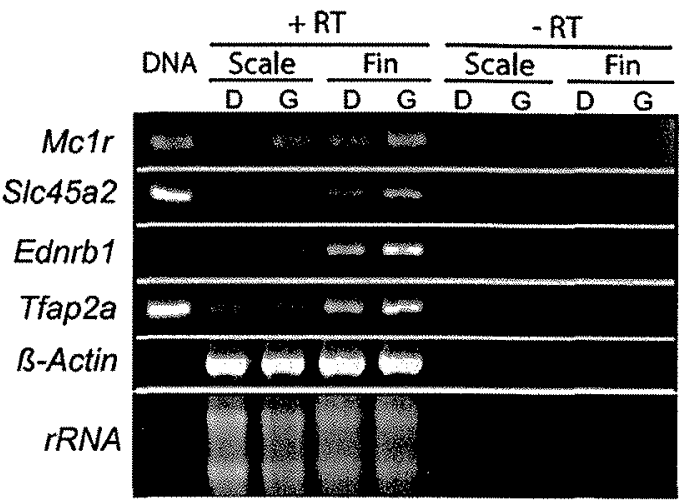

b
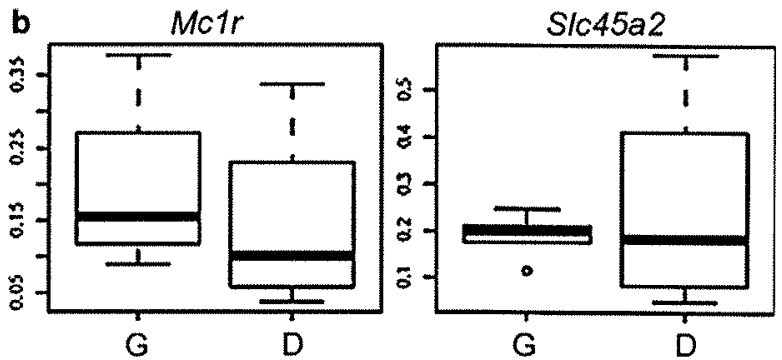

Ednrb1
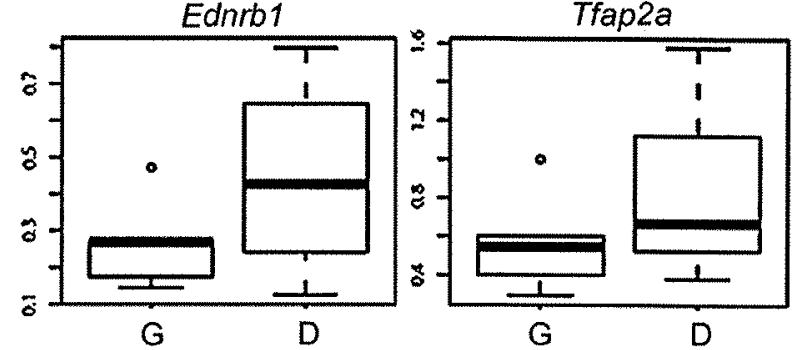

Fig. 1 a Reverse transcription PCR using candidate color genes (indicated on the left) and one control gene. The dark and gold specimens are labeled with $D$ and $G$ respectively. Quality control of cDNA produced by RT was assessed by visualizing rRNA as shown in the last row. $b$ Results of densitometric analysis comparing expression levels between gold and dark specimens. The dark line represents the median, outliers are represented by circles

other $M c l r$ sequences from other taxa, providing evidence that the correct member of the melanocortin receptor gene family was used (data not shown).

Shotgun sequencing of this $\mathrm{BAC}$ clone, yielded 27 contigs with $5 \times$ coverage, and total size of $167.634 \mathrm{bp}$ (in total $865,970 \mathrm{bp}$ ). Following gap filling and assembly, the final BAC clone had a total of $177,091 \mathrm{bp}$. It was not possible to sequence a region of approximately $300 \mathrm{bp}$ (according to agarose gel fractioning) located at position 84,793 bp between two microsatellite sequences (Fig. 2).

The sequence obtained contains a total of seven complete genes: in this order Kif7, $\mathrm{Fbln} 7, \mathrm{Mrcl}, \mathrm{Mvd}, \mathrm{Cyba}, \mathrm{Tcf} 25$, and $M c l r$ and the first exon of Def8. This synteny was found to be conserved with stickleback, medaka, fugu, and 


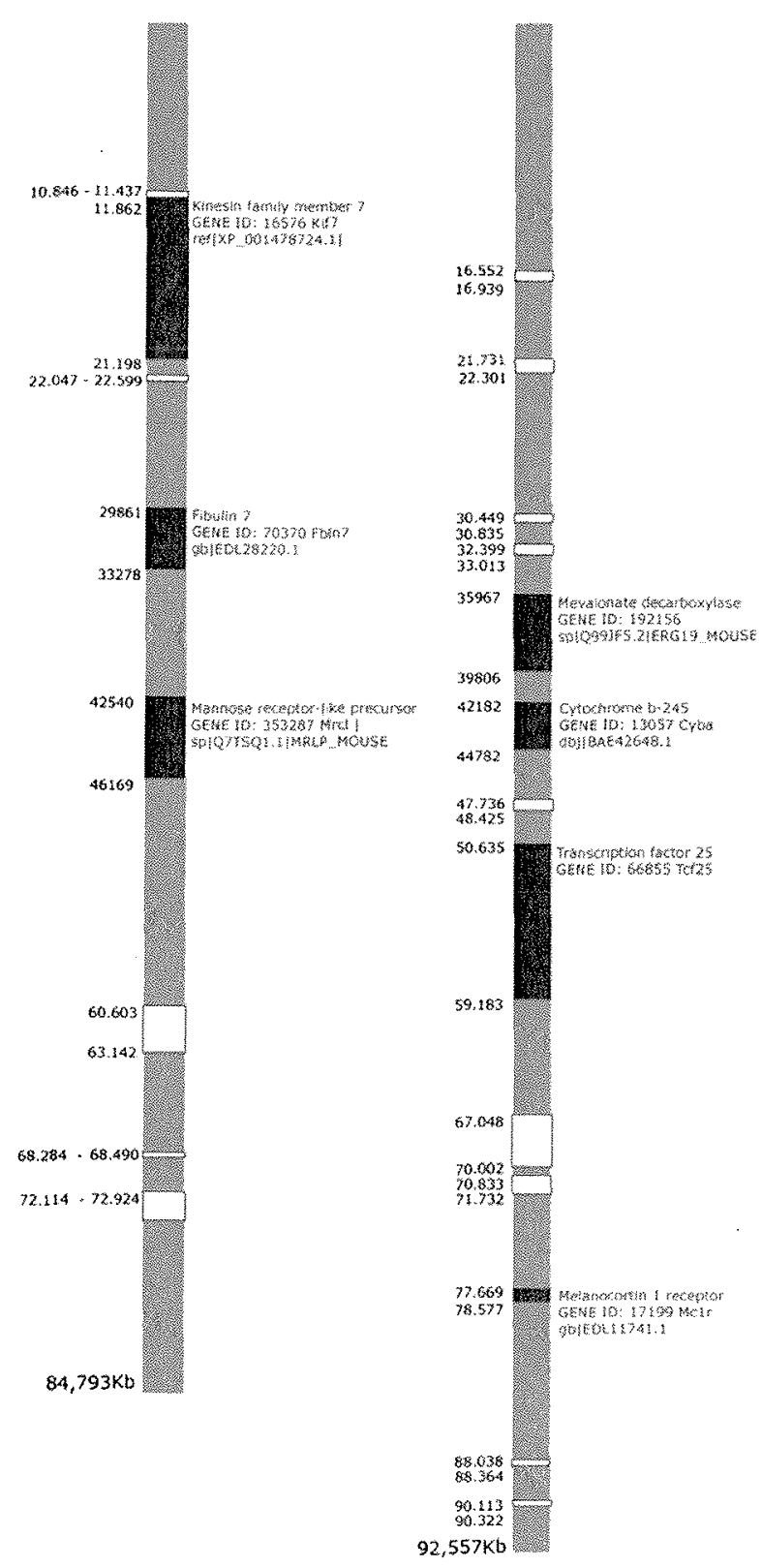

Fig. 2 Annotated BAC sequences. Genes found are shown in dark, repetitive sequences are represented in white

Tetraodon, all of which presented these orthologous genes in same order and orientation, except in zebrafish which has several differences in gene presence, order, and orientation (Fig. 3). Therefore, the zebrafish sequence was excluded from the alignment. Differences in the relative gene sizes among taxa are attributable to differences in intron size. The genes neighboring $\mathrm{Mclr}$ are conserved in all species examined, with the exception of zebrafish. This high degree of conserved synteny made it possible to align the region between the last exon of $T c f 25$ and first exon of Def8 in five teleost species including human and mouse (Fig. 4).
No polymorphisms were detected in the CNEs identified in the synteny analysis as well as the $M c l r$ coding region (see Fig. 4) ( $\approx 1,200 \mathrm{bp}$ ) sequenced for 15 gold and 15 dark field-caught specimens of A. xiloaensis. The lack of polymorphism in the sample analyzed argues that $M c l r$ is probably not the causal gene of the gold phenotype.

\section{Genetic Segregation and Population Analyses}

In order to exclude the possibility that the difference is coded by a sequence unidentified in this study, we took advantage of an available experimental cross by analyzing the segregation of the gold/dark phenotypes and of $M c 1 r$ alleles. We used $\mathrm{F}_{2}$ full-siblings obtained from one pair of $F_{1}$ hybrids between a dark female and a gold male. Consistent to a previous suggestion the gold seems to be a dominant trait (Barlow et al. 1977). Most of the $F_{I}$ fish (including the pair used for crossing) expressed the gold phenotype within 1 year. Phenotypes of the $F_{2}$ fish were distinctively dark or gold, and the ratio became very close to, but significantly different from, 1:3 even after $>4$ years of breeding $(n>200)$. We assume that some genetically gold $\mathrm{F}_{2} \mathrm{~S}$ are still remaining untransformed, but incomplete penetrance or the role of other genes or modifiers cannot be excluded at this point. All evidence so far suggests a genetic mechanism where, a dominant allele of a single locus seems to have a predominant contribution to the gold color trait.

When we compared $M c I r$ alleles of the dark and gold grandparents, a single SNP could be identified at $25 \mathrm{bp}$ upstream from the translation-initiation codon. The gold grandfather and the dark grandmother were $\mathrm{C} / \mathrm{C}$ homozygous $\mathrm{C} / \mathrm{G}$ heterozygous, respectively, and the polymorphic allele (i.e., G) was fortunately inherited to both of the F1 parents (i.e., they were $\mathrm{C} / \mathrm{G}$ heterozygous). Although this observation had already indicated that $M c 1 r$ is not the predominant contributor for the dark-gold dimorphism (see above), we genotyped $63 \mathrm{~F}_{2}$ individuals with the gold phenotype; 23,16 , and 24 of which were $\mathrm{C} / \mathrm{C}$ homozygous, $\mathrm{G} / \mathrm{G}$ homozygous, and $\mathrm{C} / \mathrm{G}$ heterozygous, respectively, showing no statistical relationship between the genotypes and the phenotypes. Considering that the segregation ratio is not significantly different from $\mathrm{C} / \mathrm{C}: \mathrm{C} / \mathrm{G}: \mathrm{G} / \mathrm{G}=1: 2: 1$ (i.e., segregation distortion was not detected, though we selected only gold $\mathrm{F}_{2} \mathrm{~s} ; P=0.077$, chi-squared test), the $M c 1 r$ locus should be inherited independently of the gold loci and have little effect on the dark-gold dimorphism.

\section{Discussion}

The color polymorphism in the Midas cichlid appears to be controlled by a single locus, in which the allele responsible for the gold phenotype is dominant. This knowledge of the 
Fig. 3 Comparative genomics of the BAC clone. The same genomic region was identified in model teleosts and mammals using the Ensembl Genome Browser and annotated manually in using Blastx. Names of genes are given on first appearance and gray lines connect the same genes



underlying genetic architecture has implications in understanding the patterns of natural variation. Color polymorphism has been shown to play a role in the sympatric population differentiation in Lake Xiloá, Nicaragua (Elmer et al. 2009). The significant difference to the expected mendelian ratios under a dominant inheritance model may indicate incomplete penetrance of the gold phenotype. This observation coupled to the known late-onset suggests that gene flow between the morphs in natural populations is higher than what would be expected based solely on matechoice experiments.
The up-regulation of Mclr in the gold morph seems at first puzzling. Since this gene is involved in melanin mobilization and synthesis and the gold phenotype is thought to result from melanophore death (Dickman et al. 1988). We expected that the expression of this gene would be correlated with the amount of melanin/melanophores and, therefore, decreased in the gold morph. It is possible that the lack of melanin or melanophores leads to increased expression of downstream genes involved in melanin synthesis and mobilization through the operation of a feedback regulation mechanism. Alternatively, the unidentified causal gene 


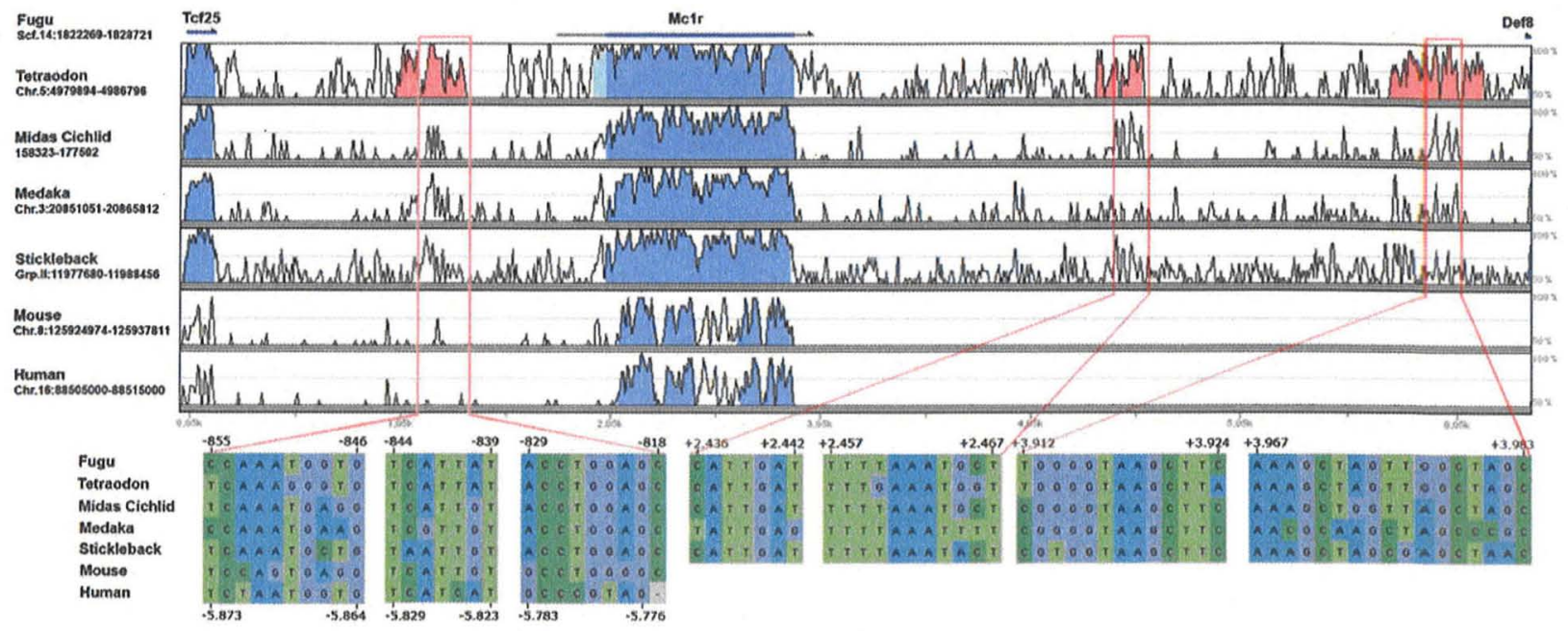

Fig. 4 Vista plot of the region between $T c f 25$ and Def8 genes that contains the $M c 1 r$ locus. CNEs are outlined and the sequences are below. The position of these regions in fugu and human genomes is given relative to the translation initiation codon

might have a downstream or regulatory effect on $M c 1 r$ expression.

Given the widespread effects of Mclr in vertebrates in general (Hoekstra 2006), we investigated the patterns of molecular variation of this locus by sequencing a BAC clone and performing comparisons between species and between the color morphs. Comparative genomic analysis revealed that synteny of the region harboring the $M c 1 r$ locus is conserved across teleosts and even to that of the two mammalian genomes included in this analysis. Mammalian genomes do, however, posses a few other genes such as Spire2, Tubb3, and Gas 8 in this genomic region that are not found in fish. The genome of medaka contains an additional gene, Afg $3 l 1$, between Def8 and Dbnddl which is located in the same region in mouse and human but is absent from the other teleosts examined. A partial sequence of $A f g 3 l l$ was found in the same region of the fugu genome, which alongside the conservation between medaka and mammals may indicate that this gene was lost from this region in the other teleosts.

The differences in gene order and orientation found in zebrafish may be related to the great phylogenetic distance between zebrafish and the other teleosts analyzed. This may imply a unique genomic history of rearrangements occurred in the lineage leading to zebrafish. Alternatively, this could simply be an artifact due to known problems with the assembly of the zebrafish genome. Either way, our results show that the genomes of many fishes retain a surprising degree of synteny across large evolutionary distances.

Comparative genomic analysis also revealed three CNEs both down- and upstream of $M c 1 r$, some of which seem to be conserved in a teleosts-specific manner. Prediction of functional regulatory elements based solely on sequence analysis has proven elusive, owing to the characteristics such as small size and tolerance for polymorphism which make them specially susceptible to false discovery (Boffelli et al. 2004). Previously identified regulatory regions of $M c l r$ in humans and mice involve the lack of a TATA box and the possible role of E-box elements to which the microphthalmia-associated transcription factor (Mitf) binds (Garcia-Borron et al. 2005). The conserved sequences identified here do not coincide with those sequences. They may, nevertheless provide good candidates for future functional studies. An interesting possibility would be the analysis of these regions in the populations of $A$. mexicanus in which the Mclr mutation was not found in the coding region (Gross et al. 2009).

The comparison of molecular variation between color morphs of the Midas cichlid revealed a lack of polymorphisms in the Mclr coding and CNEs within this population of A. xiloaensis. This species originated within crater lake Xiloá in less than 10,000 years old and it has been shown previously that intra-lake levels of polymorphism are low (Barluenga and Meyer 2004; Elmer et al. 2010). We were able to find a SNP in the mapping cross presumably because the grandparents of the cross were collected from different lakes. Since no linkage between this SNP and the color was found, we can definitely exclude the $M c 1 r$ as the causal gene for the gold coloration in the species.

Although a SNP analysis was not conducted for the other genes, which also showed differential expression in the gold morph, it seems likely that the alteration of these genes is a downstream effect. Based on a recent mapping effort, the gold locus was assigned to a genomic region that 
does not include any known candidate color gene (Fukamachi et al. in prep.).

Despite having provided spectacular examples of parallel adaptation in diverse vertebrate taxa, a role of $M c 1 r$ in adaptive evolution of fish pigmentation seems to be still wanting. To date, the involvement of $M c l r$ in pigment pattern evolution has only been reported in one teleost species (Gross et al. 2009). If the paucity of examples for the role of $\mathrm{Mclr}$ in fish coloration were upheld by further studies, one explanation for this might lie with the much larger variety of pigments and pigment cells found in fish compared to birds and mammals.

Acknowledgments This study was funded with a DFG (Deutsche Forschungsgemeinschaft) grant to AM, a Long-term Fellowship of the International Human Frontier Science Program Organization to S. F. (\#00059/2005-L), and CNPq (Brazilian National Counsel of Scientific and Technologic Development)/DAAD (Deutscher Akademischer Austausch Dienst) Ph.D grant to FH. The authors would like to thank Dr. Kathryn Elmer and the two anonymous reviewers for the critical reading and helpful suggestions.

\section{References}

Barlow GW, Rogers W, Cappeto RV (1977) Incompatibility and assortative mating in Midas cichlid. Behav Ecol Sociobiol 2:4959

Barluenga M, Meyer A (2004) The Midas cichlid species complex: incipient sympatric speciation in Nicaraguan cichlid fishes? Mol Ecol 13:2061-2076

Barluenga M, Stölting KN, Salzburger W, Muschick M, Meyer A (2006) Sympatric speciation in Nicaraguan crater lake cichlid fish. Nature 439:719-723

Boffelli D, Nobrega MA, Rubin EM (2004) Comparative genomics at the vertebrate extremes. Nat Rev Genet 5:456-465

Braasch I, Schartl M, Volff JN (2007) Evolution of pigment synthesis pathways by gene and genome duplication in fish. BMC Evol Biol 7:74

Dickman MC, Schliwa M, Barlow GW (1988) Melanophore death and disappearance produces color metamorphosis in the polychromatic Midas cichlid (Cichlasoma citrinellum). Cell Tissue Res 253:9-14

Elmer KR, Lehtonen TK, Meyer A (2009) Color assortative mating contributes to sympatric divergence of neotropical cichlid fish. Evolution 63:2750-2757

Elmer K, Kusche H, Lehtonen T, Meyer A (2010) Local variation and parallel evolution: morphological and genetic diversity across a species complex of Neotropical crater lake cichlid fishes. Phil Trans R Soc B 365:1763-1782

Endler JA (1980) Natural selection on color patterns in Poecilia reticulata. Evolution 34:76-91

Fujii R (2000) The regulation of motile activity in fish chromatophores. Pigment Cell Res 13:300-319

Fukamachi S, Shimada A, Shima A (2001) Mutations in the gene encoding $\mathrm{B}$, a novel transporter protein, reduce melanin content in medaka. Nat Genet 28:381-385
Garcia-Borron JC, Sanchez-Laorden BL, Jimenez-Cervantes C (2005) Melanocortin-1 receptor structure and functional regulation. Pigment Cell Res 18:393-410

Gross JB, Borowsky R, Tabin CJ (2009) A novel role for Mclr in the parallel evolution of depigmentation in independent population of the cavefish Astyanax mexicanus. PLoS Genet 5:e1000326

Healy E, Jordan SA, Budd PS, Suffolk R, Rees JL, Jackson IJ (2001) Functional variation of MCIR alleles from red-haired individuals. Hum Mol Genet 10:2397-2402

Hoekstra HE (2006) Genetics, development and evolution of adaptive pigmentation in vertebrates. Heredity 97:222-234

Hoekstra HE, Hirschmann RJ, Bundey RA, Insel PA, Crossland JP (2006) A single amino acid mutation contributes to adaptive beach mouse color pattern. Science 313:101-104

Kocher TD (2004) Adaptive evolution and explosive speciation: the cichlid fish model. Nat Rev Genet 5:288-298

Lalueza-Fox C, Rompler H, Caramelli D, Staubert C, Catalano G, Hughes D, Rohland N, Pilli E, Longo L, Condemi S, de la Rasilla M, Fortea J, Rosas A, Stoneking M, Schoneberg T, Bertranpetit J, Hofreiter M (2007) A melanocortin 1 receptor allele suggests varying pigmentation among Neanderthals. Science 318:1453-1455

Logan DW, Bryson-Richardson RJ, Pagán KE, Taylor MS, Currie PD, Jackson IJ (2003) The structure and evolution of the melanocortin and $\mathrm{MCH}$ receptors in fish and mammals. Genomics 81:184-191

Meyer A (1993) Phylogenetic relationships and evolutionary processes in East African cichlid fishes. Trends Ecol Evol 8:279284

Miller CT, Beleza S, Pollen AA, Schluter D, Kittles RA, Shriver MD, Kingsley DM (2007) cis-regulatory changes in kit ligand expression and parallel evolution of pigmentation in sticklebacks and humans. Cell 131:1179-1189

Mundy Nl (2005) A window on the genetics of evolution: MC1R and plumage colouration in birds. Proc Biol Sci 272:1633-1640

Parichy DM (2003) Pigment patterns: fish in stripes and spots. Curr Biol 13:R947-R950

Parichy DM (2006) Evolution of danio pigment pattern development. Heredity $97: 200-210$

Richardson J, Lundegaard PR, Reynolds NL, Dorin JR, Porteous DJ, Jackson IJ, Patton EE (2008) $m c / r$ pathway regulation of zebrafish melanosome dispersion. Zebrafish 5:289-295

Rompler H, Rohland N, Lalueza-Fox C, Willerslev E, Kuznetsova T, Rabeder G, Bertranpetit J, Schoneberg T, Hofreiter M (2006) Nuclear gene indicates coat-color polymorphism in mammoths. Science 313:62

Rosenblum EB, Hoekstra HE, Nachman MW (2004) Adaptive reptile color variation and the evolution of the Mclr gene. Evolution 58:1794-1808

Salzburger W, Braasch I, Meyer A (2007) Adaptive sequence evolution in a color gene involved in the formation of the characteristic egg-dummies of male haplochromine cichlid fishes. BMC Biol 5:1

Seehausen O, Terai Y, Magalhaes IS, Carleton KL, Mrosso HD, Miyagi R, van der Sluijs I, Schneider MV, Maan ME, Tachida H, Imai $H$, Okada $N$ (2008) Speciation through sensory drive in cichlid fish. Nature 455:620-626

Selz Y, Braasch I, Hoffmann C, Schmidt C, Schultheis C, Schartl M, Volff JN (2007) Evolution of melanocortin receptors in teleost fish: the melanocortin type I receptor. Gene 401:114-122 